\title{
Mitteilungen
}

Schwerpunktprüfung zur Erlangung

des Schwerpunktes Neonatologie

zum Facharzttitel Kinder- und

Jugendmedizin

Ort: Klinik für Neonatologie,

Universitätsspital Lausanne (CHUV)

Datum:

Schriftliche Prüfung: 4.9.2018

Mündliche Prüfung: 13.-15.11.2018

\section{Anmeldefrist: 31.7.2018}

Weitere Informationen finden Sie auf der Website des SIWF unter www.siwf.ch $\rightarrow$ Fachgebiete $\rightarrow$ Facharzttitel und Schwerpunkte (Weiterbildung) $\rightarrow$ Kinder- und Jugendmedizin

\section{Facharztprüfung}

Facharztprüfung zur Erlangung des Facharzttitels Prävention und Gesundheitswesen

Ort: Geschäftsstelle SGPG, c/o polsan, Effingerstrasse 2, Bern

Datum: Donnerstag, 13. Dezember 2018

\section{Anmeldefrist: 25. Oktober 2018}

Weitere Informationen finden Sie auf der Website des SIWF unter www.siwf.ch $\rightarrow$ Fachgebiete $\rightarrow$ Facharzttitel und Schwerpunkte (Weiterbildung) $\rightarrow$ Prävention und Gesundheitswesen

Schweizerische Ärztegesellschaft für Manuelle Medizin (SAMM)

\section{Erste schriftliche Teilprüfung}

Gemäss Fähigkeitsprogramm «Manuelle Medizin (SAMM)» vom 1. Januar 2013 müssen Kandidatinnen und Kandidaten des Fähigkeitsausweises «Manuelle Medizin (SAMM)» im Modul 4 eine erste schriftliche Teilprüfung absolvieren.

Zur Prüfung antreten darf, wer die benötigten Leistungsnachweise der Module 1-3 erbracht hat. Das erfolgreiche Bestehen der Prüfung ist Voraussetzung zur späteren Erlangung des Fähigkeitsausweises «Manuelle Medizin (SAMM)». Eine Wiederholung der Prüfung ist jedoch zweimal möglich.
Datum: Mittwoch, den 5. September 2018

\section{Zeit: $9.30 \mathrm{Uhr}$}

Ort: Centre Loewenberg, Loewenberg 43, 3280 Murten

Weitere Informationen finden Sie auf der Website der Schweizerischen Ärztegesellschaft für Manuelle Medizin (SAMM) unter www.samm.ch $\rightarrow$ Ausbildung $\rightarrow$ Anmeldungen $\rightarrow$ Erste schriftliche Teilprüfung

\section{Praktische Schlussprüfung}

Gemäss Fähigkeitsprogramm «Manuelle Medizin (SAMM)» vom 1. Januar 2013 haben Kandidatinnen und Kandidaten des Fähigkeitsausweises «Manuelle Medizin (SAMM)" nach Modul 8 eine praktische Schlussprüfung zu absolvieren. Zur Prüfung antreten darf, wer die benötigten Leistungsnachweise der Module 1-3 sowie der Module 4-8 erbracht und die erste schriftliche Teilprüfung erfolgreich absolviert hat. Das erfolgreiche Bestehen der praktischen Schlussprüfung ist Voraussetzung zur späteren Erlangung des «Fähigkeitsausweises Manuelle Medizin (SAMM)». Eine Wiederholung der Prüfung ist zweimal möglich.

Datum: Freitag, den 26. Oktober, bis Samstag, den 27. Oktober 2018

Zeit: Ca. $2 \frac{1}{2}$ Stunden am Freitag oder am Samstag

Ort: Berner Fachhochschule BFH, Fachbereich Gesundheit, Stadtbachstrasse 64, 3012 Bern

Weitere Informationen finden Sie auf der Website der Schweizerischen Ärztegesellschaft für Manuelle Medizin (SAMM) unter www.samm.ch $\rightarrow$ Ausbildung $\rightarrow$ Anmeldungen $\rightarrow$ Praktische Schlussprüfung

Schweizerische Gesellschaft für Herz- und thorakale Gefässchirurgie (SGHC)

\section{Medtronic Young Surgeons Award}

Die Schweizerische Gesellschaft für Herz- und thorakale Gefässchirurgie verleiht eine von Medtronic gesponserte, mit $20000 \mathrm{CHF}$ dotierte Auszeichnung für eine hervorragende wissenschaftliche Arbeit aus dem Gebiet der Herz- und thorakalen Gefässchirurgie.

Die vorgelegte Arbeit darf nicht für einen anderen Preis eingereicht worden sein. Sie kann als Manuskript oder als Sonderdruck vorgelegt werden. Im Falle einer bereits erfolgten Veröffentlichung darf diese nicht länger als sechs Monate zurückliegen.

Bewerber dürfen noch keinen Facharzttitel für Herz- und thorakale Gefässchirurgie haben und müssen in der Schweiz angestellt sein.

Der Preis muss für die Ausbildung verwendet werden und wird erst nach Vorlegen des Verwendungszweckes ausbezahlt.

Bewerber reichen ihre Arbeit in vierfacher Ausführung bis 1. April 2018 dem Sekretär der Schweizerischen Gesellschaft für Herz- und thorakale Gefässchirurgie ein:

Prof. Dr. med. Christoph Huber, Chef du Service de Chirurgie Cardio-Vasculaire, HUG, Rue Gabrielle-Perret-Gentil 4, 1205 Genève

Prof. M. Genoni, Präsident SGHC Prof. Dr. med. Chr. Huber, Sekretär SGHC

Gesellschaft für die Geschichte der Schweizer Psychiatrie und Psychotherapie (GGSP)

Preis für Schweizer Psychiatriegeschichte

Die Gesellschaft für die Geschichte der Schweizer Psychiatrie und Psychotherapie (GGSP) lobt zweijährlich einen Preis für Arbeiten zur Schweizer Psychiatriegeschichte aus.

\section{Eingereichte Arbeiten}

Eingereicht werden können wissenschaftliche Originalarbeiten in der Form von Monographien, Beiträgen zu Sammelwerken oder Zeitschriftenartikel, die in den letzten zwei Jahren veröffentlicht wurden oder deren Publikation bereits schriftlich bestätigt wurde. Monographien muss eine zwei- bis fünfseitige Zusammenfassung beigelegt werden. Die Arbeiten können in deutscher, französischer oder englischer Sprache eingereicht werden.

\section{Angesprochene Autorinnen und Autoren}

Wir möchten besonders Nachwuchswissenschaftler/-innen im Bereich der Psychiatriegeschichte dazu ermutigen, ihre Arbeiten einzureichen.

Preissumme

Die Preissumme beträgt

1000 CHF für eine Arbeit von einer einzelnen Autorin/einem einzelnen Autor, 
1500 CHF für Arbeiten mit mehreren Autorinnen/Autoren.

\section{Vorgehensweise}

Die Arbeit muss elektronisch bis spätestens am 30. April 2018 an folgende E-Mail eingereicht werden: paul.hoff[at]puk.zh.ch

Die Beurteilung findet durch ein interdisziplinäres Komitee statt, worauf sämtliche Teilnehmenden über den Entscheid informiert werden.

Die Preisträgerin / der Preisträger wird an die Jahresversammlung der GGSP vom 30. August 2018 eingeladen, wo sie/er die Arbeit in einem Vortrag vorstellt und den Preis entgegennimmt.

Wir freuen uns auf Ihre Bewerbung!

Prof. Dr. med. Dr. phil. Paul Hoff, Präsident GGSP
Schweizerische Gesellschaft für Neuroradiologie SGNR

\section{Peter Huber Preis 2018}

Die Schweizerische Gesellschaft für Neuroradiologie schreibt zum Gedächtnis an Herrn Professor Dr. med. Peter Huber und zur Förderung des Fachgebietes der Neuroradiologie den Peter Huber Preis für eine experimentelle und/oder klinische Arbeit aus dem Gesamtgebiet der Neuroradiologie (allgemeine diagnostische Neuroradiologie, funktionelle Neuroradiologie, interventionelle Neuroradiologie, pädiatrische Neuroradiologie, Neuroradiologie der Kopf- und Halsregion) aus.

Der Erstautor sollte in neuroradiologischer Weiterbildung stehen oder eine neuroradiologische Weiterbildung abgeschlossen haben und nicht über 40 Jahre alt sein. Die eingereichte Arbeit darf 50 Seiten nicht überschreiten und muss in englischer oder deutscher Sprache verfasst sein. Die Arbeit sollte noch nicht ausgezeichnet worden sein und darf nicht an anderer Stelle zur Prämierung eingereicht sein. Pro Person kann nur eine Arbeit eingereicht werden.

Der gestiftete Preis ist mit 2000 CHF dotiert und wird im Rahmen der Jahrestagung vom 24. und 25. Mai 2018 in Lugano verliehen. Der Preisträger/die Preisträgerin wird zwei Wochen vor dem Kongress über die Entscheidung des Preiskomitees benachrichtigt und soll die Ergebnisse der Arbeit in einer 4-minütigen Zusammenfassung vorstellen. Der Preis ist teilbar.

Die Arbeiten sind per Mail (mit Abbildungen) bis zum 30. März 2018 an die Geschäftsstelle der Schweizerischen Gesellschaft für Neuroradiologie SGNR, c/o IMK Institut für Medizin und Kommunikation AG, einzureichen (sgnr[at]imk.ch).

Für den Vorstand der SGNR Prof. Dr. med. Luca Remonda, Präsident

Aktuelles Thema auf unserer Website -
«Ein Register schafft Klarheit und Sicherheit»
Weshalb es nun an der Zeit ist, auch in der Schweiz über die Einführung
der vermuteten Zustimmung bei Organspenden nachzudenken.

